\title{
P01-2-18
}

Poster session

\section{Betulin attenuates atherosclerosis in apoE-/- mice}

\author{
Yiping Wang, Yuzhou Gui, Hong Yan, Fei Gao, Cong Xi
}

\section{State Key Laboratory of Drug Research, Shanghai Institute of Materia Medica, China}

Aim: Betulin is a pentacyclic triterpenoid isolated from the bark of yellow and white birch trees. In this study we examined the effects of betulin on atherosclerosis in apoE-/- mice and the underlying mechanisms.

Methods: Murine macrophage RAW264.7 cells and human monocyte-derived THP-1 cells were tested. Cholesterol efflux was assessed using $\left[{ }^{3} \mathrm{H}\right]$-cholesterol efflux assay. The expression of ATP-binding cassette transporter A1 and G1 (ABCA1 and ABCG1) was examined using RT-PCR and Western-blotting. The ABCA1 promoter activity was evaluated using luciferase activity assay. Male apoE-/- mice fed on a high-fat-diet (HFD), and received betulin (20 and $40 \mathrm{mg} / \mathrm{kg} / \mathrm{d}$, ig) for 12 weeks. The macrophage content and ABCA1 expression in the aortic sinuses were evaluated with immunofluorescence staining. The hepatic, intestinal and fecal cholesterol were also analyzed in the mice.

Results: In RAW264.7 cells, betulin (0.1-2.5 $\mu \mathrm{g} / \mathrm{mL})$ dose-dependently ameliorated oxLDL-induced cholesterol accumulation and enhanced cholesterol efflux. In both RAW264.7 and THP-1 cells, betulin increased the expression of ABCA1 and ABCG1 via suppressing the transcriptional repressors sterol-responsive element-binding proteins (SREBPs) that bound to E-box motifs in ABCA1 promoter, whereas E-box binding site mutation markedly attenuated betulininduced ABCA1 promoter activity. In HFD-fed apoE-/- mice, betulin administration significantly reduced lesions in en face aortas and aortic sinuses. Furthermore, betulin administration significantly increased ABCA1 expression and suppressed macrophage positive areas in the aortic sinuses. Moreover, betulin administration improved plasma lipid profiles and enhanced fecal cholesterol excretion in the mice.

Conclusion: Betulin attenuates atherosclerosis in apoE-/- mice by promoting cholesterol efflux in macrophages. 